\title{
Front Matter: Volume 7554
}

, "Front Matter: Volume 7554," Proc. SPIE 7554, Optical Coherence Tomography and Coherence Domain Optical Methods in Biomedicine XIV, 755401 (22 March 2010); doi: 10.1117/12.855911

SPIE. Event: SPIE BiOS, 2010, San Francisco, California, United States 


\section{PROGRESS IN BIOMEDICAL OPTICS AND IMAGING}

\section{Optical Coherence Tomography and Coherence Domain Optical Methods in Biomedicine XIV}

Joseph A. Izatt

James G. Fujimoto

Valery V. Tuchin

Editors

25-27 January 2010

San Francisco, United States

Sponsored and Published by

SPIE

Volume 7554

Proceedings of SPIE, 1605-7422, v. 7554

SPIE is an international society advancing an interdisciplinary approach to the science and application of light. 
The papers included in this volume were part of the technical conference cited on the cover and title page. Papers were selected and subject to review by the editors and conference program committee. Some conference presentations may not be available for publication. The papers published in these proceedings reflect the work and thoughts of the authors and are published herein as submitted. The publisher is not responsible for the validity of the information or for any outcomes resulting from reliance thereon.

Please use the following format to cite material from this book:

Author(s), "Title of Paper," in Optical Coherence Tomography and Coherence Domain Optical Methods in Biomedicine XIV, edited by Joseph A. Izatt, James G. Fujimoto, Valery V. Tuchin, Proceedings of SPIE Vol. 7554 (SPIE, Bellingham, WA, 2010) Article CID Number.

ISSN 1605-7422

ISBN 9780819479501

Published by

SPIE

P.O. Box 10, Bellingham, Washington 98227-0010 USA

Telephone +1 3606763290 (Pacific Time) · Fax +1 3606471445

SPIE.org

Copyright @ 2010, Society of Photo-Optical Instrumentation Engineers.

Copying of material in this book for internal or personal use, or for the internal or personal use of specific clients, beyond the fair use provisions granted by the U.S. Copyright Law is authorized by SPIE subject to payment of copying fees. The Transactional Reporting Service base fee for this volume is $\$ 18.00$ per article (or portion thereof), which should be paid directly to the Copyright Clearance Center (CCC), 222 Rosewood Drive, Danvers, MA 01923. Payment may also be made electronically through CCC Online at copyright.com. Other copying for republication, resale, advertising or promotion, or any form of systematic or multiple reproduction of any material in this book is prohibited except with permission in writing from the publisher. The CCC fee code is 1605 $7422 / 10 / \$ 18.00$.

Printed in the United States of America.

Publication of record for individual papers is online in the SPIE Digital Library.

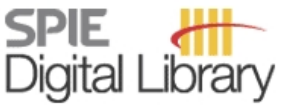

SPIEDigitallibrary.org

Paper Numbering: Proceedings of SPIE follow an e-First publication model, with papers published first online and then in print and on CD-ROM. Papers are published as they are submitted and meet publication criteria. A unique, consistent, permanent citation identifier (CID) number is assigned to each article at the time of the first publication. Utilization of CIDs allows articles to be fully citable as soon they are published online, and connects the same identifier to all online, print, and electronic versions of the publication. SPIE uses a six-digit CID article numbering system in which:

- The first four digits correspond to the SPIE volume number.

- The last two digits indicate publication order within the volume using a Base 36 numbering system employing both numerals and letters. These two-number sets start with 00, 01, 02, 03, 04, $05,06,07,08,09,0 A, 0 B \ldots 0 Z$, followed by 10-1Z, 20-2Z, etc.

The CID number appears on each page of the manuscript. The complete citation is used on the first page, and an abbreviated version on subsequent pages. Numbers in the index correspond to the last two digits of the six-digit CID number. 


\section{Contents}

xi Conference Committee

xiii Introduction

\section{SESSION 1 CARDIOVASCULAR APPLICATIONS}

755403 System design and image processing algorithms for frequency domain optical coherence tomography in the coronary arteries [7554-02]

D. C. Adler, C. XU, C. Petersen, J. M. Schmitt, LightLab Imaging Inc. (United States)

755406 Investigations of the intravascular backscattering distribution of light in optical coherence tomography [7554-05]

P. Cimalla, J. Walther, E. Koch, Dresden Univ. of Technology (Germany)

\section{SESSION 2 ENDOSCOPIC AND CANCER IMAGING}

755408 In vivo early detection of smoke-induced airway injury using 3-dimensional swept source optical coherence tomography [7554-07]

J. Yin, Univ. of California, Irvine (United States); G. Liu, J. Zhang, L. Yu, S. Mahon, D. Mukai, Beckman Laser Institute (United States); M. Brenner, Beckman Laser Institute (United States) and Univ. of California, Irvine (United States); Z. Chen, Univ. of California, Irvine (United

States) and Beckman Laser Institute (United States)

755409 Multiscale imaging of human thyroid pathologies using integrated optical coherence tomography (OCT) and optical coherence microscopy (OCM) [7554-08]

C. Zhou, Massachusetts Institute of Technology (United States); Y. Wang, Beth Israel Deaconess Medical Ctr., Harvard Medical School (United States) and Montefiore Medical Ctr. and Albert Einstein Medical School (United States); A. D. Aguirre, Massachusetts Institute of Technology (United States) and Harvard-MIT Division of Health Sciences and Technology (United States); T. Tsai, Massachusetts Institute of Technology (United States); D. W. Cohen,

J. L. Connolly, Beth Israel Deaconess Medical Ctr., Harvard Medical School (United States);

J. G. Fujimoto, Massachusetts Institute of Technology (United States)

\section{SESSION 3 OPHTHALMIC: NEW TECHNOLOGY}

7554 OE Adaptive optics spectral domain optical coherence tomography with one-micrometer light source [7554-13]

K. Kurokawa, K. Sasaki, S. Makita, Y. Yasuno, Univ. of Tsukuba (Japan) and Computational Optics and Ophthalmology Group (Japan)

7554 OF Real-time intraoperative spectral domain optical coherence tomography for vitreoretinal surgery [7554-14]

Y. K. Tao, Duke Univ. (United States); C. A. Toth, Duke Univ. Medical Ctr. (United States);

J. A. Izatt, Duke Univ. (United States) 
7554 ol In vitro retinal imaging with full field swept source optical coherence tomography [7554-17] J. Fergusson, Cardiff Univ. (United Kingdom); B. Považay, B. Hofer, W. Drexler, Medical Univ. of Vienna (Austria)

7554 0J Variable lateral size imaging of the human retina in vivo by combined confocal/en face optical coherence tomography with closed loop OPD-locked low coherence interferometry based active axial eye motion tracking [7554-18]

R. G. Cucu, Univ. of Kent (United Kingdom); M. W. Hathaway, OTI/Opko (Canada);

A. Gh. Podoleanu, Univ. of Kent (United Kingdom); R. B. Rosen, New York Eye and Ear Infirmary (United States)

7554 OK Ultra-high speed full range complex spectral domain optical coherence tomography for volumetric imaging at 140,000 A scans per second [7554-19]

H. M. Subhash, L. An, R. K. Wang, Oregon Health \& Science Univ. (United States)

\section{SESSION 4 CELLULAR AND SMALL ANIMAL IMAGING}

7554 ON Mutant and wild type cell chemotaxis in 3D and 4D with ultrahigh-resolution optical coherence tomography [7554-22]

S. M. Rey, Cardiff Univ. (United Kingdom); B. Považay, B. Hofer, A. Unterhuber, Medical Univ. of Vienna (Austria) and Cardiff Univ. (United Kingdom); A. Harwood, Cardiff Univ. (United Kingdom); W. Drexler, Medical Univ. of Vienna (Austria) and Cardiff Univ. (United Kingdom)

$7554 \mathrm{OP} \quad$ A heterodyne Mach-Zehnder Interferometer employing static and dynamic phase demodulation techniques for live-cell imaging [7554-24]

S. Joseph, Univ. of Limerick (Ireland); J.-M. Gineste, M. Whelan, European Commission Joint Research Ctr. (Italy); D. Newport, Univ. of Limerick (Ireland)

7554 OS In vivo optical coherence tomography of percutaneous implants in hairless mice [7554-27] S. Donner, Laser Zentrum Hannover e.V. (Germany) and Hannover Medical School (Germany); F. Witte, Hannover Medical School (Germany) and Univ. of Pittsburgh (United States); I. Bartsch, Hannover Medical School (Germany); F. Petraglia, Univ. of Pittsburgh (United States); O. Massow, M. Heidrich, H. Lubatschowski, Laser Zentrum Hannover e.V. (Germany); A. Heisterkamp, A. Krüger, Laser Zentrum Hannover e.V. (Germany) and Hannover Medical School (Germany)

7554 OT Characterizing matrix remodeling in collagen gels using optical coherence tomography [7554-28]

D. Levitz, M. T. Hinds, S. R. Hanson, S. L. Jacques, Oregon Health \& Science Univ. (United States)

\section{SESSION 5 OTHER CLINICAL APPLICATIONS}

7554 OV Optimizing penetration depth, contrast, and resolution in 3D dermatologic OCT [7554-30] A. Aneesh, Cardiff Univ. (United Kingdom); B. Považay, B. Hofer, Medical Univ. Vienna (Austria); E. Z. Zhang, Univ. College London (United Kingdom); C. Kendall, Gloucestershire Hospital (United Kingdom); J. Laufer, Univ. College London (United Kingdom); S. Popov, Imperial College (United Kingdom); C. Glittenberg, S. Binder, Ludwig Boltzmann Institute (Austria); N. Stone, Gloucestershire Hospital (United Kingdom); P. C. Beard, Univ. College London (United Kingdom); W. Drexler, Medical Univ. Vienna (Austria) and Cardiff Univ. (United Kingdom) 
7554 0X Design of a dual-modality imaging system using optical coherence tomography and fluorescence lifetime imaging microscopy for anatomical and biochemical diagnosis of tissue [7554-32]

S. Shrestha, J. A. Jo, J. Park, P. Pande, B. E. Applegate, Texas A\&M Univ. (United States)

$7554 \mathrm{OZ}$ Guidance of hard tissue ablation by forward-viewing optical coherence tomography [7554-34]

P. J. L. Webster, B. Y. C. Leung, Queen's Univ. (Canada); V. X. D. Yang, Univ. of Toronto

(Canada), Ryerson Univ. (Canada), and Sunnybrook Health Science Ctr. (Canada);

J. M. Fraser, Queen's Univ. (Canada)

\section{SESSION 6 DOPPLER OCT}

755412 Observation of blood optical inhomogeneity using joint spectral and time domain OCT [7554-37]

D. Bukowska, A. Szkulmowska, I. Grulkowski, S. Tamborski, M. Szkulmowski, Nicolaus

Copernicus Univ. (Poland); R. Leitgeb, Univ. of Vienna (Austria); A. Kowalczyk, M. Wojtkowski, Nicolaus Copernicus Univ. (Poland)

755413 BM-mode scanning with parabolic phase modulation for full range Doppler optical tomography [7554-38]

F. Jaillon, Univ. of Tsukuba (Japan); S. Makita, Y. Yasuno, Univ. of Tsukuba (Japan) and Computational Optics and Ophthalmology Group (Japan)

755414 Real-time bulk motion insensitive flow segmentation algorithm for Doppler spectral optical coherence tomography [7554-39]

M. Szkulmowski, A. Szkulmowska, D. Szlag, I. Grulkowski, A. Kowalczyk, M. Wojtkowski,

Nicolaus Copernicus Univ. (Poland)

\section{SESSION 7 OCT NEW TECHNOLOGY}

755417 Coherent transfer functions and extended depth of field [7554-42]

M. Villiger, C. Pache, Ecole Polytechnique Fédérale de Lausanne (Switzerland);

R. A. Leitgeb, Medical Univ. of Vienna (Austria); T. Lasser, Ecole Polytechnique Fédérale de Lausanne (Switzerland)

755418 Simultaneous 6-channel optical coherence tomography using a high-power telescope-less polygon-based swept laser in dual-amplifier configuration [7554-43]

M. K. K. Leung, A. Mariampillai, Univ. of Toronto (Canada); B. A. Standish, Ryerson Univ. (Canada); K. K. C. Lee, I. A. Vitkin, Univ. of Toronto (Canada); V. X. D. Yang, Ryerson Univ. (Canada)

7554 1B Integrated photonic circuit in silicon on insulator for Fourier domain optical coherence tomography [7554-46]

G. Yurtsever, P. Dumon, W. Bogaerts, R. Baets, Ghent Univ. (Belgium) 
7554 lE Frequency comb swept lasers for optical coherence tomography [7554-49]

T.-H. Tsai, C. Zhou, D. Adler, J. G. Fujimoto, Massachusetts Institute of Technology (United States)

7554 IF Compact ultrafast reflective Fabry-Perot tunable lasers for OCT imaging applications [7554-50]

M. Kuznetsov, W. Atia, B. Johnson, D. Flanders, Axsun Technologies Inc. (United States)

7554 1H FDML swept source at 1060 nm using a tapered amplifier [7554-52]

S. Marschall, Technical Univ. of Denmark (Denmark); T. Klein, W. Wieser, B. Biedermann, Ludwig-Maximilians-Univ. München (Germany); K. Hsu, Micron Optics, Inc. (United States); B. Sumpf, K.-H. Hasler, G. Erbert, Ferdinand-Braun-Institut für Höchstfrequenztechnik (Germany); O. B. Jensen, C. Pedersen, Technical Univ. of Denmark (Denmark); R. Huber, Ludwig-Maximilians-Univ. München (Germany); P. E. Andersen, Technical Univ. of Denmark (Denmark)

7554 II Ultra broadband Fourier domain mode locked swept source based on dual SOAs and WDM couplers [7554-53]

J. Zhang, G. Liu, Z. Chen, Beckman Laser Institute (United States) and Univ. of California, Irvine (United States)

7554 i Multiband swept laser source for frequency domain optical coherence tomography [7554-54]

J. Jiang, Univ. of Kansas (United States) and Tianjin Univ. (China); R. Hui, Univ. of Kansas (United States)

\section{SESSION 9 PSOCT}

$75541 \mathrm{M} \quad$ Polarization sensitive optical coherence tomography of melanin provides tissue inherent contrast based on depolarization [7554-57]

B. Baumann, Medical Univ. of Vienna (Austria); S. O. Baumann, T. Konegger, Vienna Univ. of Technology (Austria); M. Pircher, E. Götzinger, H. Sattmann, Medical Univ. of Vienna (Austria); M. Litschaver, Vienna Univ. of Technology (Austria); C. K. Hitzenberger, Medical Univ. of Vienna (Austria)

$75541 \mathrm{~N} \quad$ Full range polarization-sensitive swept-source optical coherence tomography at $1 \mu \mathrm{m}$ with polarization modulation and BM-mode scan [7554-58]

M. Yamanari, S. Makita, Y. H. Lim, Y. Yasuno, Univ. of Tsukuba (Japan) and Computational Optics and Ophthalmology Group (Japan)

755410 Single camera polarization sensitive spectral domain OCT by spatial frequency encoding [7554-59]

T. Schmoll, E. Goetzinger, M. Pircher, C. K. Hitzenberger, R. A. Leitgeb, Medical Univ. Vienna (Austria)

$75541 Q \quad$ High-speed spectral domain polarization-sensitive OCT using a single InGaAs line-scan camera and an optical switch [7554-61]

S.-W. Lee, H.-W. Jeong, B.-M. Kim, Korea Univ. (Korea, Republic of) 
7554 IR Ultrahigh-resolution fiber-based polarization sensitive spectral domain optical coherence tomography [7554-62]

E. Götzinger, M. Pircher, B. Baumann, C. K. Hitzenberger, Medical Univ. of Vienna (Austria)

7554 is From controlling the shape of Talbot bands' visibility to improving the sensitivity decay with depth in FD-OCT [7554-63]

A. Gh. Podoleanu, M. Hughes, A. Bradu, D. Woods, Univ. of Kent (United Kingdom)

7554 1V Non-harmonic analysis for high-resolution optical coherence tomography [7554-66]

C. Chong, Santec Corp. (Japan); X. Cao, Univ. of Toyama (Japan); A. Morosawa, K. Totsuka,

T. Suzuki, Santec Corp. (Japan); S. Hirobayashi, Univ. of Toyama (Japan)

7554 1W Optical coherence tomography resolution improvement by step-frequency encoding [7554-67]

E. Bousi, I. Charalambous, C. Pitris, Univ. of Cyprus (Cyprus)

$75541 \mathrm{~T} \quad$ Twofold improvement in axial resolution of optical coherence tomography by four-pass sample probing [7554-68]

M. Sylwestrzak, E. A. Kwiatkowska, P. Targowski, Nicolaus Copernicus Univ. (Poland)

\section{SESSION 11 NOVEL CONTRAST MECHANISMS}

755412 Pump-probe optical coherence microscopy [7554-70]

Q. Wan, B. E. Applegate, Texas A\&M Univ. (United States)

755420 Magnetomotive optical coherence elastography for relating lung structure and function in cystic fibrosis [7554-71]

R. K. Chhetri, J. Carpenter, R. Superfine, S. H. Randell, A. L. Oldenburg, The Univ. of North Carolina at Chapel Hill (United States)

755421 Overcoming barriers in topical administration of gold nanoparticles for optical coherence tomography using multimodal delivery [7554-72]

C. S. Kim, Univ. of California, Irvine (United States) and Beckman Laser Institute (United States); P. Wilder-Smith, Y.-C. Ahn, L.-H. L. Liaw, Beckman Laser Institute (United States);

Z. Chen, Univ. of California, Irvine (United States) and Beckman Laser Institute (United

States); Y. J. Kwon, Univ. of California, Irvine (United States)

755424 Monitoring small changes in blood hematocrit using phase sensitive spectral domain optical coherence tomography [7554-75]

V. G. R. Manne, R. K. Manapuram, N. Sudheendran, Univ. of Houston (United States);

K. V. Larin, Univ. of Houston (United States) and Saratov State Univ. (Russian Federation)

\section{SESSION 12 FULL FIELD/OCM/PHASE CONTRAST}

755425 Dark-field optical coherence microscopy [7554-77]

C. Pache, M. L. Villiger, T. Lasser, Ecole Polytechnique Fédérale de Lausanne (Switzerland) 
755427 Crosstalk rejection in full-field optical coherence tomography using spatially incoherent illumination with a partially coherent source [7554-79]

A. Dhalla, J. Migacz, J. A. Izatt, Duke Univ. (United States)

755429 Low-coherence enhanced backscattering imaging with simultaneous multiple spatial filters [7554-81]

J. Liu, Z. Xu, Y. L. Kim, Purdue Univ. (United States)

7554 2B The role of a detector dead time in phase-resolved Doppler analysis using spectral domain optical coherence tomography [7554-83]

J. Walther, P. Cimalla, E. Koch, Univ. of Technology Dresden (Germany)

7554 2C Sub-cellular resolution imaging with Gabor domain optical coherence microscopy [7554-84]

P. Meemon, CREOL, The College of Optics and Photonics, Univ. of Central Florida (United

States); K. S. Lee, The Institute of Optics, Univ. of Rochester (United States); S. Murali, General Optics (Asia) Ltd. (India); I. Kaya, CREOL, The College of Optics and Photonics, Univ. of Central Florida (United States); K. P. Thompson, Optical Research Associates (United States); J. P. Rolland, CREOL, The College of Optics and Photonics, Univ. of Central Florida (United States) and The Institute of Optics, Univ. of Rochester (United States)

\section{POSTER SESSION}

7554 2D In vivo 3D FD OCT of subpleural lung parenchyma in the intact thorax [7554-85]

S. Meissner, C. Schnabel, L. Knels, E. Koch, Univ. of Technology Dresden (Germany)

$75542 \mathrm{E} \quad$ Optical Doppler tomography and spectral Doppler imaging of localized ischemic stroke in a mouse model [7554-86]

L. Yu, E. Nguyen, G. Liu, B. Rao, B. Choi, Z. Chen, Beckman Laser Institute (United States)

$75542 \mathrm{~F} \quad$ Monitoring of sutured flexor tendons using spectral domain optical coherence tomography [7554-87]

C.-M. B. Tay, T.-H. Chow, B.-K. Ng, Nanyang Technological Univ. (Singapore); M. He, W.-T. A. Gan, K.-S. A. Chong, National Univ. Hospital (Singapore)

7554 2G Preliminary optical coherence tomography investigation of the temporo-mandibular joint disc [7554-88]

C. Mărcăuteanu, E. Demjan, C. Sinescu, M. Negrutiu, A. Motoc, R. Lighezan, L. Vasile, Univ. de Medicina si Farmacie Victor Babes, Timisoara (Romania); M. Hughes, A. Bradu, G. Dobre, A. G. Podoleanu, Univ. of Kent (United Kingdom)

$75542 \mathrm{H} \quad$ A study on the qualitative morphological features of the muscle and subcutaneous shapes in vivo using Fourier-domain common path OCT [7554-89]

J.-H. Han, L. Xuan, J. U. Kang, Johns Hopkins Univ. (United States); C. G. Song, Johns Hopkins Univ. (United States) and Chonbuk National Univ. (Korea, Republic of)

75542 Clinical optical coherence tomography combined with multiphoton tomography for evaluation of several skin disorders [7554-90]

K. König, JenLab GmbH (Germany) and Saarland Univ. (Germany); M. Speicher, R. Bückle, J. Reckfort, JenLab GmbH (Germany); G. McKenzie, Michelson Diagnostics Ltd. (United Kingdom); J. Welzel, General Hospital Augsburg (Germany); M. J. Koehler, P. Elsner, M. Kaatz, Friedrich-Schiller-Univ. Jena (Germany) 
$75542 \mathrm{~L} \quad$ Morphometric analysis of the optic nerve head with optical coherence tomography [7554-93]

M. Young, S. Lee, E. Gibson, Simon Fraser Univ. (Canada); K. Hsu, Micron Optics (United States); M. F. Beg, Simon Fraser Univ. (Canada); P. J. Mackenzie, Univ. of British Columbia (Canada); M. V. Sarunic, Simon Fraser Univ. (Canada)

7554 2M Dynamic analysis of mental sweating by optical coherence tomography [7554-94] M. Ohmi, M. Tanigawa, H. Saigusa, A. Yamada, Y. Ueda, M. Haruna, Osaka Univ. (Japan)

$75542 \mathrm{~N}$ Minimal invasive localization of the germinal disc in ovo for subsequent chicken sexing using optical coherence tomography [7554-95]

A. Burkhardt, S. Geißler, P. Cimalla, J. Walther, E. Koch, Univ. of Technology Dresden (Germany)

755420 High-speed concatenation of frequency ramps using sampled grating distributed Bragg reflector laser diode sources for OCT resolution enhancement [7554-96]

B. George, D. Derickson, California Polytechnic State Univ. (United States)

$75542 \mathrm{P} \quad$ Tunable semiconductor laser based on interaction between strongly mismatched Fabry-Perot interferometer and waveguide modes [7554-97]

A. A. Moiseev, G. V. Gelikonov, Institute of Applied Physics (Russian Federation);

E. A. Mashcovitch, Nizjniy Novgorod State Univ. (Russian Federation); V. M. Gelikonov, Institute of Applied Physics (Russian Federation)

$75542 Q \quad$ MEMS scanner based swept-source laser for optical coherence tomography [7554-98] K. Totsuka, K. Isamoto, T. Sakai, A. Morosawa, C. Chong, Santec Corp. (Japan)

$75542 S$ Real-time display on SD-OCT using a linear-in-wavenumber spectrometer and a graphics processing unit [7554-100]

Y. Watanabe, T. Itagaki, Yamagata Univ. (Japan)

7554 2T Frequency domain optical coherence tomography with subsequent depth resolved spectroscopic image analysis [7554-101]

C. Kasseck, Ruhr-Univ. Bochum (Germany); V. Jaedicke, Georg Agricola Univ. of Applied Sciences (Germany); N. C. Gerhardt, Ruhr-Univ. Bochum (Germany); H. Welp, Georg Agricola Univ. of Applied Sciences (Germany); M. R. Hofmann, Ruhr-Univ. Bochum (Germany)

$75542 \mathrm{U}$ Adaptive filtering of optical coherent tomography fringe data with ensemble empirical mode decomposition [7554-102]

G. Liu, Beckman Laser Institute (United States) and Univ. of California, Irvine (United States);

J. Zhang, Beckman Laser Institute (United States); L. Yu, Z. Chen, Beckman Laser Institute (United States) and Univ. of California, Irvine (United States)

$75542 \mathrm{~V} \quad$ High speed full range imaging with harmonic detection swept source optical coherence tomography [7554-103]

C. Huang, S. M. Massick, K. A. Peterson, Southwest Sciences, Inc. (United States);

A. B. Vakhtin, Vista Photonics 
7554 2W Signal processing with unequally spaced data in Fourier-domain optical coherence tomography [7554-104]

S. Vergnole, D. Lévesque, National Research Council Canada (Canada); S. S. Sherif, Univ. of Manitoba (Canada); G. Lamouche, National Research Council Canada (Canada)

7554 2X Evaluation of complex conjugate artifact removal methods used in spectrometer-based Fourier-domain optical coherence tomography systems: a comparative study [7554-105] D. Y. Kim, J. S. Werner, Univ. of California, Davis Medical Ctr. (United States) and Univ. of California, Davis (United States); R. J. Zawadzki, Univ. of California, Davis Medical Ctr. (United States)

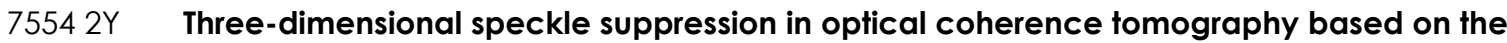
curvelet transform [7554-106]

L. Yu, Z. Jian, B. Rao, B. J. Tromberg, Z. Chen, Beckman Laser Institute (United States)

$75542 Z$ Multi-beam resolution video-rate swept-source optical coherence tomography (OCT) provides endogenous contrast for in vivo blood flow independent of flow direction [7554-107]

F. Bazant-Hegemark, D. Woods, S. Hattersley, J. Holmes, Michelson Diagnostics Ltd. (United Kingdom)

$755430 \quad$ Focusing light through living tissue [7554-108]

I. M. Vellekoop, C. M. Aegerter, Univ. of Zürich (Switzerland)

755431 Coherent noise compensation improvement in spectral-domain optical coherence tomography [7554-109]

G. V. Gelikonov, V. M. Gelikonov, I. V. Kasatkina, D. A. Terpelov, P. A. Shilyagin, Institute of Applied Physics (Russian Federation)

755432 Effective bandwidth in spectral-domain OCT [7554-1 10]

M. Jiang, S. Jiao, The Univ. of Southern California (United States)

755434 Speckle imaging by combination of mathematical morphology and contrast ratio [7554-112]

Z. Li, W. Xie, L. Fan, S. Cai, H. Li, Fujian Normal Univ. (China)

Author Index 


\title{
Conference Committee
}

\author{
Symposium Chairs \\ James G. Fujimoto, Massachusetts Institute of Technology (United \\ States) \\ R. Rox Anderson, Wellman Center for Photomedicine, Massachusetts \\ General Hospital (United States) and Harvard School of Medicine \\ (United States)
}

Program Track Chairs

Tuan Vo-Dinh, Duke University (United States)

Anita Mahadevan-Jansen, Vanderbilt University (United States)

Conference Chairs

Joseph A. Izatt, Duke University (United States)

James G. Fujimoto, Massachusetts Institute of Technology (United States)

Valery V. Tuchin, Saratov State University (Russian Federation)

Program Committee

Peter E. Andersen, Danmarks Tekniske Universitet (Denmark)

Stephen A. Boppart, University of Illinois at Urbana-Champaign (United States)

Zhongping Chen, University of California, Irvine (United States)

Johannes F. de Boer, Vrije Universiteit Amsterdam (Netherlands)

Wolfgang Drexler, Cardiff University (United Kingdom)

Christoph K. Hitzenberger, Medizinische Universität Wien (Austria)

Martin J. Leahy, University of Limerick (Ireland)

Rainer A. Leitgeb, Universität Wien (Austria)

Xingde Li, The Johns Hopkins University (United States)

Adrian Gh. Podoleanu, University of Kent (United Kingdom)

Andrew M. Rollins, Case Western Reserve University (United States)

Natalia M. Shakhova, Institute of Applied Physics (Russian Federation)

Guillermo J. Tearney, Massachusetts General Hospital (United States)

Ruikang Wang, Oregon Health \& Science University (United States)

Maciej Wojtkowski, Nicolaus Copernicus University (Poland)

Yoshiaki Yasuno, University of Tsukuba (Japan) 
Session Chairs

1 Cardiovascular Applications

Joseph A. Izatt, Duke University (United States)

2 Endoscopic and Cancer Imaging

James G. Fujimoto, Massachusetts Institute of Technology (United States)

3 Ophthalmic: New Technology

Maciej Wojtkowski, Nicolaus Copernicus University (Poland)

$4 \quad$ Cellular and Small Animal Imaging

Andrew M. Rollins, Case Western Reserve University (United States)

5 Other Clinical Applications

Wolfgang Drexler, Cardiff University (United Kingdom)

6 Doppler OCT

Rainer A. Leitgeb, Medizinische Universitat Wien (Austria)

7 OCT New Technology

Guillermo J. Tearney, Wellman Center for Photomedicine (United States)

8 Swept Light Source New Technology

Zhongping Chen, University of Illinois at Urbana-Champaign (United States)

9 PSOCT

Christoph K. Hitzenberger, Medizinische Universität Wien (Austria)

10 Signal/Image Processing

Ruikang Wang, Oregon Health \& Science University (United States)

11 Novel Contrast Mechanisms

Stephen A. Boppart, University of Illinois at Urbana-Champaign (United States)

12 Full Field/OCM/Phase Contrast

Valery V. Tuchin, Saratov State University (Russian

Federation) 


\section{Introduction}

These proceedings are from the Coherence Domain Optical Methods and Optical Coherence Tomography in Biomedicine XIV conference held January 2527, 2010 at the SPIE Photonics West Symposia in San Francisco, California. This year's conference featured 111 oral and poster presentations from leading national and international research groups.

The conference was organized into several sessions including: Cardiovascular Applications; Endoscopic and Cancer Imaging; Ophthalmic New Technology; Cellular and Small Animal Imaging; Other Clinical Applications; Doppler OCT; OCT New Technology; Swept Light Source New Technology; PSOCT; Signal/Image Processing; Novel Contrast Mechanisms; Full Field/OCM/Phase Contrast, and a poster session with 28 presentations. As usual, a predominant fraction of the papers focused on optical coherence tomography - basic research, instrumentation and applications.

This year there were significant advances in clinical and biological applications (five sessions: Cardiovascular Applications; Endoscopic and Cancer Imaging; Ophthalmic New Technology; Cellular and Small Animal Imaging; Other Clinical Applications, with 34 oral and 11 poster presentations) with discussion of OCT technologies for studying coronary arteries including the natural history of coronary atherosclerotic plaques, real-time monitoring of cardiac RF-ablation lesion formation, monitoring of shear stress in the developing heart tube, in vivo early detection of airway injury, in vivo tomography of sub-pleural lung parenchyma in the intact thorax, multi-scale imaging of thyroid pathologies, in vivo 3D-imaging of swine esophagus, clinical study of the biliary tree, differentiating carcinogenesis stages of oral lesions, real-time monitoring of vitreoretinal surgery, choroidal neovascularization and retinal imaging, keratometry, studying cell chemotaxis and dynamics, live rat embryos and muscular dystrophy in mouse model imaging, monitoring of sutured flexor tendons and temporomandibular joint disc, monitoring of skin disease, the nerve fiber and optic nerve head imaging, noninvasive assessment of biofilm growth in the middle ear, guidance of hard tissue ablation, and for some other fields of clinical application.

Six oral presentations in the session on Doppler OCT reported advanced technologies for speckle mediated tracking of tumor angiogenesis and response to vascular targeted PDT, optical micro-angiography in brain trauma, full range Doppler optical tomography, and high-resolution wide-field blood perfusion mapping of retina and choroid. The influence of blood optical inhomogeneity on Doppler OCT signal was also discussed. Real-time bulk motion insensitive flow segmentation algorithms for Doppler spectral OCT were presented as well. 
The session on OCT New Technologies contained eight oral papers and was devoted to further development of OCT components and whole systems, such as Fourier-domain mode-locked (FDML)-based multi-spot OCT at 4,100,000 A-scans and 4 Gvoxels per second, multichannel OCT using a high-power telescope-less polygon-based swept laser in dual amplifier configuration, a novel sample arm for dynamic optical coherence elastography, large field OCT by optical movement tracking of a single point probe, integrated photonic circuit in silicon on insulator for FD OCT, and novel CW stable and high-power supercontinuum light source.

A special session on Swept Light Source New Technology contained eight oral presentations with discussion of frequency comb swept lasers, compact ultrafast reflective Fabry-Perot tunable lasers, FDML lasers - high frequency driving vs. buffering, ultra-broadband FDML swept source based on dual SOAs and WDM couples, fiber-based swept source at $1060 \mathrm{~nm}$ using tapered amplifier, swept source system based on integrated thermo-optic tunable laser chip, and wavelength swept amplified spontaneous emission source at $1060 \mathrm{~nm}$ with $\mathrm{Yb}$ doped fiber post-amplification.

This year's Polarization-Sensitive OCT (PSOCT) session with six oral presentations highlighted the following actual problems: melanin inherent contrast based on depolarization and PSOCT FD imaging based on depolarized of light, and described a number of advanced systems: full range PS swept-source OCT at 1 $\mu \mathrm{m}$ with polarization modulation and BM-mode scan, single camera PS spectraldomain OCT by spatial frequency encoding, high-speed spectral-domain PS OCT using a single InGaAs line-scan camera and an optical switch, and ultrahighresolution fiber-based PS spectral-domain OCT.

The Signal/Imaging Processing session presented six oral papers on controlling the shape of Talbot bands' visibility to improving the sensitivity decay with depth in FD-OCT, performance of reduced bit-depth acquisition for FD-OCT, sonification of OCT data and images, non-harmonic analysis for high-resolution OCT, resolution improvement in OCT by step-frequency encoding, and twofold improvement in axial resolution of OCT by four-pass sample probing.

The Novel Contrast Mechanisms session contained seven oral presentations and discussed magnetomotive OCT for in vivo molecular imaging of mammary tumor using targeted magnetic nanoprobes and for elastographic relating lung structure and function in cystic fibrosis, the development of pump-probe OCM, monitoring of multimodal gold nanoparticle delivery, tissue differentiation in human lymph nodes using parameterized OCT, reconstruction of absorption profile of ICG using spectral OCT, and monitoring small changes in blood hematocrit using phase-sensitive spectral-domain OCT.

The last session on Full Field/OCM/Phase Contrast with eight oral papers presented a number of techniques and applications including dark-field OCM, ultrahigh speed phase mapping (512,000 A-scan rate) line field FD OCT, crosstalk 
rejection in full-field OCT using spatially incoherent illumination with a partially coherent source, low-coherence enhanced backscattering imaging with multiple spatial filters, double common-path phase microscopy, breast cancer surgery and full-field OCT in the operative room, and sub-cellular resolution imaging with Gabor-domain OCM.

The poster session contained 28 papers on the major topics mentioned above.

A short course for engineers, scientists, and clinicians; SC312: Principles and Applications of Optical Coherence Tomography by James Fujimoto accompanied the conference.

All submissions were fully peer reviewed. Authors were requested to submit a 3page summary of their paper. The program committee evaluated the submissions for technical content and assigned a numerical score to each paper. The selection of the papers as oral presentations, posters, or non-acceptance was based upon the program committee score. We have had very positive feedback and a record number of submissions and attendees again this year.

The conference chairs would like to thank the members of the technical program committee for their help in organizing the conference. We sincerely appreciate the support of the SPIE and the conference staff. Finally, we would like to thank all of the conference attendees and manuscript authors for their contributions and participation which helped to make this meeting a success.

\author{
Joseph A. Izatt \\ James G. Fujimoto \\ Valery V. Tuchin
}


Downloaded From: https://www.spiedigitallibrary.org/conference-proceedings-of-spie on 26 Apr 2023

Terms of Use: https://www.spiedigitallibrary.org/terms-of-use 